\title{
A NEW APPROACH FOR PREISACH DISTRIBUTION FUNCTION IDENTIFICATION USING FEW EXPERIMENTAL DATA
}

\author{
Leila CHELGHOUM, Fatma Zohra LOUAI, Nasreddine NAIT-SAID \\ Department of Electrical engineering, Faculty of technology, University Hadj Lakhdar of Batna, E-mail: chel_lei@yahoo.fr
}

\begin{abstract}
In this paper, we describe a new numerical method for Preisach distribution function identification. This approach is characterized from other identification methods published in the literature by the use of few experimental data extracted from first magnetization curve(usually provided by the manufacturers). In order to entirely discretize the Preisach triangle, the new method uses a cloud of points created and positioned in $(H-M)$-plane with respect to the experimental data. Mathematical developments revealed a parameter denoted $\lambda$ used in the positioning and whose variation affects significantly the magnetic properties. Numerical simulations performed for different values of this parameter have shown its great influence on the hysteresis loops shape. Comparisons of the obtained results with experimental data allowed the identification of the $\lambda$ factor as the ratio of the magnetization at the bend saturation to the magnetization of saturation. The efficiency and applicability of the developed method have been tested through numerical simulations and comparisons with available experimental data.
\end{abstract}

Keywords: Hysteresis modeling, Preisach density function, identification method, Preisach model, first magnetization curve, created points, magnetic materials.

\section{INTRODUCTION}

The magnetic hysteresis observed in ferromagnetic materials has been a long standing problem and still attracting the attention of many researchers [1], [2], [3]. Experimental observations show that hysteresis is a phenomenon resulting from the combined action of several identical interacting entities. In ferromagnetic materials, the magnetization is a result of the interaction of magnetic Weiss domains [4]. Several mathematical models of hysteresis have been developed in order to describe magnetic materials behavior and their properties [1], [4], [5], [6]. Among these models, the Preisach model is one of the most powerful tools in modeling magnetic hysteresis. The implementation of this model is closely related to the identification of its density function. Several identification methods have been published in the literatures and are mainly based on two approaches: analytical approaches and the numerical approaches [1], [5], [6], [7], [8], [9]. In the following, after a brief presentation of the principle of hysteresis modeling using the Preisach model, we present our new approach for identifying the Preisach density function using few experimental data extracted from the experimental first magnetization curve. Mathematical developments revealed a parameter denoted $\lambda$ characterizing the ferromagnetic material. Finally, we have validated our numerical results by comparisons with available experimental data.

\section{THE SCALAR PREISACH MODEL}

The Preisach model describes hysteresis as a weighting sum of many elemental hysteresis operators $\gamma_{(\beta, \alpha)}$ with parameters $\alpha, \beta(\alpha \geq \beta)$ taken as upper and lower switching values, respectively. Output of elemental operators $\gamma_{(\beta, \alpha)}$ would be only +1 or -1 (see Fig. 1(a)).

Parallel to the concept of elementary hysteresis operators introduced, a statistical function representing their spatial distribution in the Preisach triangle (see Fig. 1(b)) must be determined. This function is called Preisach density function [4] and is denoted $v(\alpha, \beta)$ with $v(\alpha, \beta) \geq 0$. Its analytical or numerical determination is based on experimental data. If an infinite set of the hysteresis operators are considered and according to the Preisach model the magnetization $M(t)$ can be expressed as follows:

$$
M(t)=\iint_{\alpha \geq \beta} v(\alpha, \beta) \gamma_{(\alpha, \beta)}[H(t)] d \alpha d \beta
$$

where $M(t)$ is the output of the model (the magnetization) at state $t$ and $H(t)$ is the input (applied magnetic field) at the same state.

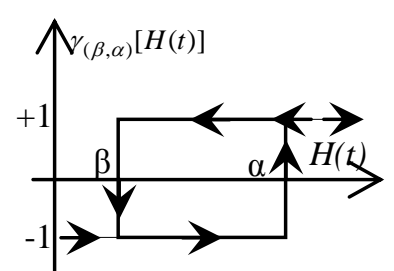

(a)

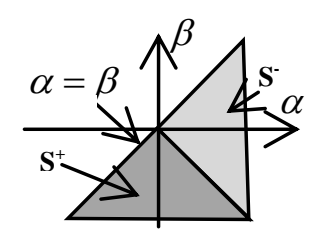

(b)
Fig. 1 (a): Elementary hysteresis operator $\gamma(\beta, \alpha)$, (b): Preisach triangle. 


$$
\begin{gathered}
\text { Since } \\
\begin{cases}\gamma_{(\beta, \alpha)}[H(t)]=+1 & \text { if }(\alpha, \beta) \in S^{+}(t) \\
\gamma_{(\beta, \alpha)}[H(t)]=-1 & \text { if }(\alpha, \beta) \in S^{-}(t)\end{cases}
\end{gathered}
$$

Thus, at any instant of time the integral in (1) can be subdivided into two integrals over $\mathrm{S}^{+}(\mathrm{t})$ and $\mathrm{S}^{-}(\mathrm{t})$ :

$$
M(t)=\iint_{S^{+}(t)} v(\alpha, \beta) d \alpha d \beta-\iint_{S^{-}(t)} v(\alpha, \beta) d \alpha d \beta
$$

\section{PREISACH DENSITY FUNCTION IDENTIFICATION}

The Preisach density function identification is a critical step in the simulation of hysteresis by using the Preisach model. Several approaches have been proposed in the literature: analytical approaches generally based on the choice of the density function shape [6] and numerical approaches that require experimental data [1], [4], [7], [9]. Subsequently, we will briefly explain the link between the curve $(H, M)$ and the Preisach triangle.

Starting from a value $\left(-H_{s}\right)$ of the excitation, we increase it monotonically until the value $\left(H_{1}=\alpha_{1}\right)$, we obtain the part of the magnetization curve shown in (see Fig. 2.(a)). Then, we decrease the excitation to the value $\left(H_{2}=\beta_{1}\right)$ and we obtain the part of the magnetization curve shown in (see Fig. 2.(b)). Finally, we obtain the cell denoted by $T\left(\alpha_{1}, \beta_{1}\right)$ (see Fig. 2.(b)) which represents the variation $\Delta M$ between two different states $\left(\alpha_{1}, M \alpha_{1}\right)$ and $\left(\beta_{1}, M \alpha_{1 \beta 1}\right)$.

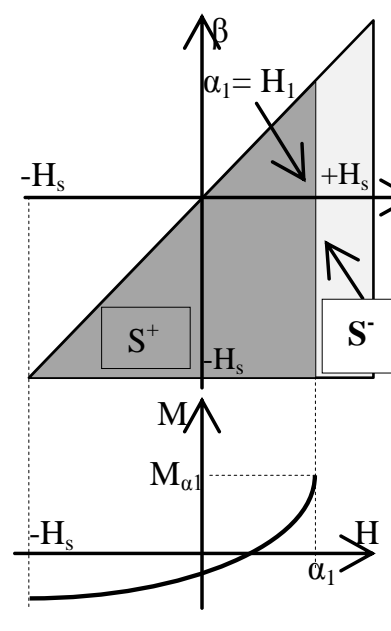

(a)



(b)
Fig. 2 (a),(b): respectively an increase and a decrease of the magnetization in the $(H, M)$-plane and their Preisach triangle representations.

Subsequently, the variation is given by [1]:

$$
\Delta M=\left(M_{\alpha 1}-M_{\alpha 1 \beta 1}\right)=2 \iiint_{T(\alpha 1, \beta 1)} v(\alpha, \beta) d \alpha d \beta
$$

The above equation is a bijective relationship between Preisach triangle discretization (second term of (4)) and the magnetization variations (first term of (4)). It should also be noted that each cell of the discretized Preisach triangle provides information about the discretized value of the Preisach density function.

Based on these observations, we develop a new identification method using few experimental data extracted from first magnetization curve. These experimental points are associated with a cloud of created points whose magnetization is bounded by the saturation values. These created points are placed in the $(H, M)$ plane by following a developed procedure and they allow full Preisach triangle discretization.

\section{FORMULATION OF THE NEW PROPOSED IDENTIFICATION METHOD}

In the identification process, we make the following assumptions:

1. in each cell $C_{(i, j)}$ of the discretized Preisach triangle, the density function $v(\alpha, \beta)$ is constant:

$$
\iint_{C_{(i, j)}} v(\alpha, \beta) d \alpha d \beta=S_{(i, j)} v_{(i, j)}=v_{i j}
$$

where $S_{(i, j)}$ is the area of the cell $C_{(i, j)}, v_{(i, j)}$ the discrete value of the Preisach density function and $v_{\mathrm{ij}}$ the discrete value multiplied by the area of the cell.

2 . symmetry of the density function with respect to the line $(\alpha=-\beta)$ in the Preisach triangle.

Using these assuptions, we consider:

- $(p)$ given experimental points $\left[\left(-H_{i},-M_{i}\right), i=1, p\right]$ extracted from first magnetization curve (see Fig. 3.(a)) with $\Delta H$ constant.

$\Delta H=H_{i+1}-H_{i}$

- ( $p$ ) symmetrical points of the given experimental points relative to the $(H, M)$-plane origin and noted [(-Hi, $-M i), i=1, p$ ] (see Fig. 3.(a)).

- a cloud of $\left(p^{2}\right)$ created points noted by $\left(H_{i j}{ }^{*}, M_{i j}{ }^{*}\right)$ and defined in the $(H, M)$-plane by:

$$
\left\{\begin{array}{l}
-H_{s}<H_{i j}^{*}<+H_{s} \\
-M_{s}<M_{i j}^{*}<M_{f m c}(H)
\end{array}\right.
$$

where $M_{f m c}(H)$ is the first magnetization curve (completed by its symmetrical relative to the origin $(0,0)$ ). $H_{s}$ and $M_{s}$ are respectively the saturation field and the magnetization of saturation given by the first magnetization curve.

Created points are arranged in the $(H, M)$-plane following the procedure described below:

1. horizontal positioning: for the $\mathrm{k}^{\text {th }}$ experimental point $\left(H_{k}, M_{k}\right)$, we define $\left[\left(H_{k j}{ }^{*}, M_{k j}{ }^{*}\right), j=1,2 k-1\right]$ created points positioned in the region of the $(H, M)$-plane delimited by the lines $\left(H=H_{k}\right)$ and $\left(H=-H_{k}\right)$ : 


$$
\left\{\begin{array}{l}
-H_{k}<H_{k j}^{*}<+H_{k} \\
\Delta H^{*}=\Delta H=H_{k j+1}^{*}-H_{k j}^{*}
\end{array}\right.
$$

It should be noted that created points are also equally spaced (second equation of (8)).

2. vertical positioning: The magnetization value $M_{k j}^{*}$ is determined using a grandeur denoted by $\delta M_{k}$ which represents the magnetization variation between two successive experimental points. The factor $\lambda$ is used and it allows vertical positioning of the created point $\left(H_{k j}^{*}, M_{k j}^{*}\right)$. Subsequently, for each experimental point, we obtain $(p-k)$ created points arranged vertically below it. The conditions given by (7) imply that the factor $\lambda$ must be positive. After positioning all the created points in the $(H, M)$-plane, we apply the Preisach model technique (4). Taking into account the assumption number 2 (symmetry of the density function), we consider only $p(p+1)$ cells in the discretized Preisach triangle.

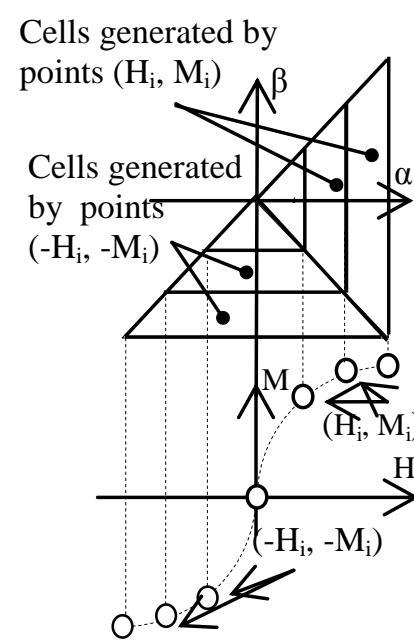

(a)

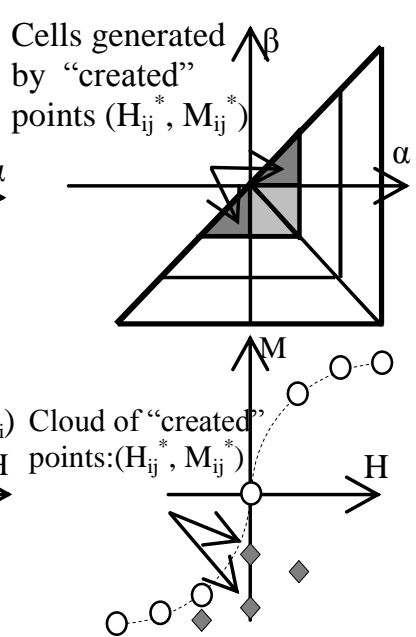

(b)
Fig. 3 Geometric interpretation: (a) Preisach triangle discretization by considering given experimental points and their symmetrical (b) Preisach triangle discretization by considering created points on the

To explain the process used in the new proposed identification method, we consider $p=3$ experimental points extracted from a given first magnetization curve and their symmetrical relative to the origin of the $(H, M)$ plane: $\left(-H_{3},-M_{3}\right),\left(-H_{2},-M_{2}\right),\left(-H_{l},-M_{1}\right),\left(H_{0}, M_{0}\right),\left(H_{l}, M_{l}\right)$, $\left(H_{2}, M_{2}\right),\left(H_{3}, M_{3}\right)$.

For the first experimental point $\left(H_{1}, M_{1}\right)$ and its symmetrical $\left(-H_{1},-M_{1}\right)$ (see Fig. 4$)$, in order to identify the unknown content of the cell $\mathrm{C}_{11}$ (corresponding to the unknown value $v_{11}$ of discrete density function) we use a created point $\left(H_{11}^{*}, M_{11}^{*}\right)$ arranged vertically under the point $\left(H_{0}=0, M_{0}=0\right)$ ( see Fig. 4 ) and defined as:

$\left\{\begin{array}{l}-M_{1}<M_{11}^{*}<M_{1}<0 \\ H_{11}^{*}=0\end{array}\right.$

The magnetization value $M_{11}^{*}$ is determined using the grandeur denoted by $\delta M_{1}$ representing the magnetization variation of the magnetization between the experimental points $\left[\left(-H_{1},-M_{1}\right),\left(H_{0}, M_{0}\right)\right]$ and the positioning factor $\lambda$ which allows here the positioning of the created point $\left(H_{11}^{*}, M_{11}^{*}\right)$ with respect to the two considered experimental points:

$\left\{\begin{array}{l}\delta M_{1}=-M_{1}-\left(-M_{0}\right) \\ M_{11}^{*}=M_{0}-\lambda \delta M_{1}\end{array}\right.$

As the magnetization variation between $-M_{1}$ and $M_{11}^{*}$ involves only the cell $C_{(1,1)}$ of the discretized Preisach triangle (Fig. 4) and by using (4), we have:

$M_{11}^{*}-\left(-M_{1}\right)=2 \iint_{C_{(1,1)}} v(\alpha, \beta) d \alpha d \beta=2 v_{11}$

Subsequently, we can determine the value of the distribution function at the cell $\mathrm{C}_{(1,2)}$ (Fig. 4):

$$
\left(+M_{1}\right)-M_{11}^{*}=2 \iint_{C_{(1,1)}+C_{(1,2)}} v(\alpha, \beta) d \alpha d \beta=2\left(v_{11}+v_{12}\right)
$$

For each considered experimental point, we construct a system of equations whose unknowns are the discrete value of the Preisach distribution function. For this, magnetization variations given by equation (4) will be denoted by $\Delta M_{(m)}^{(k)}$, where the indices $(k)$ and $(m=1, \ldots, 2 k)$ represent respectively the number of the considered experimental point and the number of equations generated by the application of the developed procedure.

For the first experimental point $(k=1)$ and $(m=1,2)$; we have 2 equations:

$$
\left\{\begin{array}{l}
\Delta M_{(1)}^{(1)}=M_{11}^{*}-\left(-M_{1}\right)=2 v_{11} \\
\Delta M_{(2)}^{(1)}=M_{1}-M_{11}^{*}=2 v_{12}+\Delta M_{(1)}^{(1)}
\end{array}\right.
$$

At this step, we have determined the discrete values of the distribution function $\left(v_{11}, v_{12}\right)$. 




Fig. 4 Geometric interpretation of the procedure for the first experimental point $\left(\mathrm{H}_{1}, \mathrm{M}_{1}\right)$.

For the second experimental point $\left(H_{2}, M_{2}\right)$, we use 3 created points $\left(H_{21}^{*}, M_{21}^{*}\right), \quad\left(H_{22}^{*}, M_{22}^{*}\right)$ and $\left(H_{23}^{*}, M_{23}^{*}\right)$ positioned vertically respectively under the points $\left(-H_{1},-M_{1}\right),\left(H_{11}^{*}, M_{11}^{*}\right),\left(H_{1}, M_{1}\right)$ and defined by:

$$
\begin{aligned}
& \left\{\begin{array}{l}
\delta M_{2}=-M_{2}-\left(-M_{1}\right) \\
M_{21}^{*}=-M_{1}-\lambda \delta M_{2} \\
M_{22}^{*}=-M_{11}^{*}-\lambda \delta M_{2} \\
M_{23}^{*}=M_{1}-\lambda \delta M_{2}
\end{array}\right. \\
& \left\{\begin{array}{l}
\Delta M_{(1)}^{(2)}=M_{21}^{*}-\left(-M_{2}\right)=2 v_{22} \\
\Delta M_{(2)}^{(2)}=M_{22}^{*}-M_{21}^{*}=2 v_{23}+\Delta M_{(1)}^{(1)} \\
\Delta M_{(3)}^{(2)}=M_{23}^{*}-M_{22}^{*}=2 v_{24}+\Delta M_{(2)}^{(1)} \\
\Delta M_{(4)}^{(2)}=\left(M_{2}\right)-M_{23}^{*}=2 \sum_{j=2}^{5} v_{2 j}
\end{array}\right.
\end{aligned}
$$

For the third experimental point $\left(H_{3}, M_{3}\right)$ and its symmetrical $\left(-H_{3},-M_{3}\right)$ relative to the origin of the $(H, M)$-plane, we use 5 created points $\left(H_{31}^{*}, M_{31}^{*}\right)$, $\left(H_{32}^{*}, M_{32}^{*}\right),\left(H_{33}^{*}, M_{33}^{*}\right),\left(H_{34}^{*}, M_{34}^{*}\right)$ and $\left(H_{35}^{*}, M_{35}^{*}\right)$ positioned vertically respectively under the points $\left(-H_{2},-M_{2}\right),\left(H_{21}^{*}, M_{21}^{*}\right),\left(H_{22}^{*}, M_{22}^{*}\right)$,

$\left(H_{23}^{*}, M_{23}^{*}\right),\left(H_{2}, M_{2}\right)$ and defined by: $\left\{\begin{array}{l}\delta M_{3}=-M_{3}-\left(-M_{2}\right) \\ M_{31}^{*}=-M_{2}-\lambda \delta M_{3} \\ M_{32}^{*}=-M_{21}^{*}-\lambda \delta M_{3} \\ M_{33}^{*}=-M_{22}^{*}-\lambda \delta M_{3} \\ M_{34}^{*}=-M_{23}^{*}-\lambda \delta M_{3} \\ M_{35}^{*}=+M_{2}-\lambda \delta M_{3}\end{array}\right.$

Here also we obtain the following algebraic system:

$$
\left\{\begin{array}{l}
\Delta M_{(1)}^{(3)}=M_{31}^{*}-\left(-M_{3}\right)=2 v_{33} \\
\Delta M_{(2)}^{(3)}=M_{32}^{*}-M_{31}^{*}=2 v_{34}+\Delta M_{(1)}^{(2)} \\
\Delta M_{(3)}^{(3)}=M_{33}^{*}-M_{32}^{*}=2 v_{35}+\Delta M_{(2)}^{(2)} \\
\Delta M_{(4)}^{(3)}=M_{34}^{*}-M_{33}^{*}=2 v_{36}+\Delta M_{(3)}^{(2)} \\
\Delta M_{(5)}^{(3)}=M_{35}^{*}-M_{34}^{*}=2 v_{37}+\Delta M_{(4)}^{(2)} \\
\Delta M_{(6)}^{(3)}=\left(M_{3}\right)-M_{35}^{*}=2 \sum_{k=3}^{8} v_{3 k}
\end{array}\right.
$$

Finally, if we consider $(p)$ experimental points, we obtain a system of $p(p+1)$ equations whose unknowns are the discrete values of the density function. Numerical solution provides discrete values of $v_{i j}$.

\section{NUMERICAL RESULTS}

For the validation of the proposed identification method, we have developed a numerical simulation code based on the following steps:

1. Recovery of the magnetic properties of the material (first magnetization curve).

2. Choice of the $\lambda$ factor by using first magnetization curve.

3. Positioning in the $(H, M)$-plane of the "created" points relative to the considered experimental points by using a specific developed algorithm.

4. Application of the developed technique for the Preisach triangle discretization and algebraic systems generation.

5. Systems resolution and obtaining of discrete values of the distribution function.

6. Representation of the numerical hysteresis loop and comparison with the experimental hysteresis loop.

For numerical simulations, we considered 16 experimental points extracted from the first magnetization curve of a soft magnetic alloy (Fe-Si). Following the procedure described above, we obtain a system of 272 equations solved numerically. Simulations were carried

with different values of the factor $\lambda(\lambda=0.5, \lambda=1.0$, $\lambda=1.5, \lambda=0.01)$.

\section{For $\lambda=1 / 2$ :}




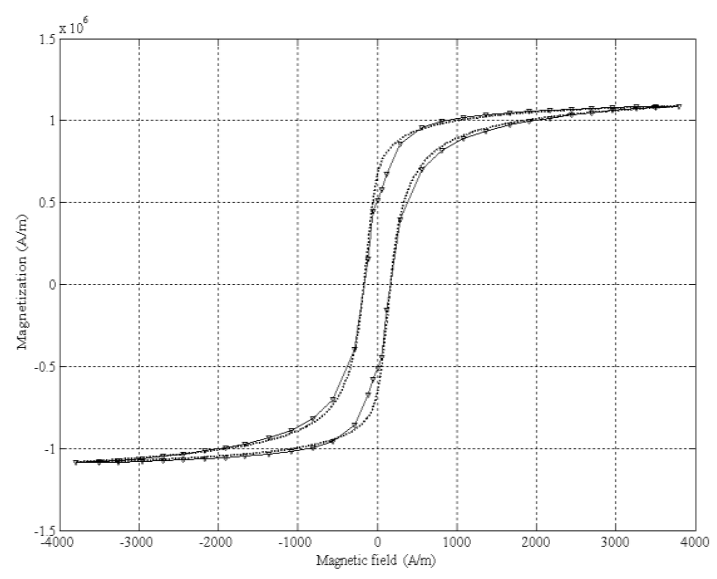

(a)

Fig. 5 Experimental measured curve (dotted line) and simulated curve (solid line with marker) generated by the proposed method.

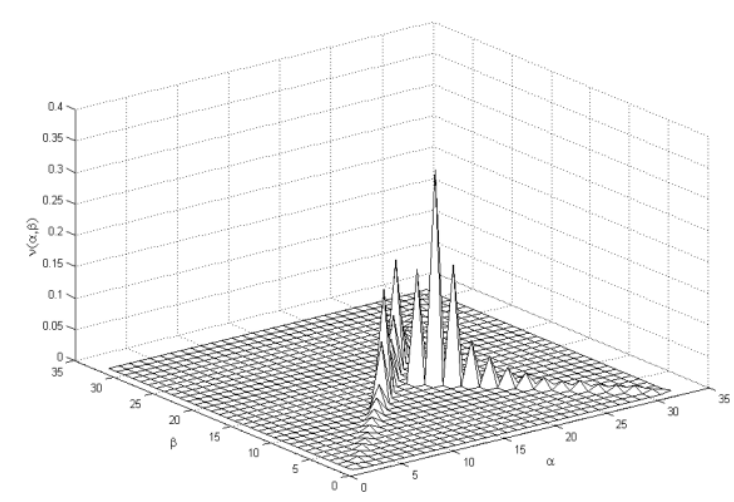

(b)

Fig. 6 Preisach distribution function generated by the proposed method.

2. For $\lambda=1$ :



(a)

Fig. 7 Experimental measured curve (dotted line) and simulated curve (solid line with marker) generated by the proposed method.

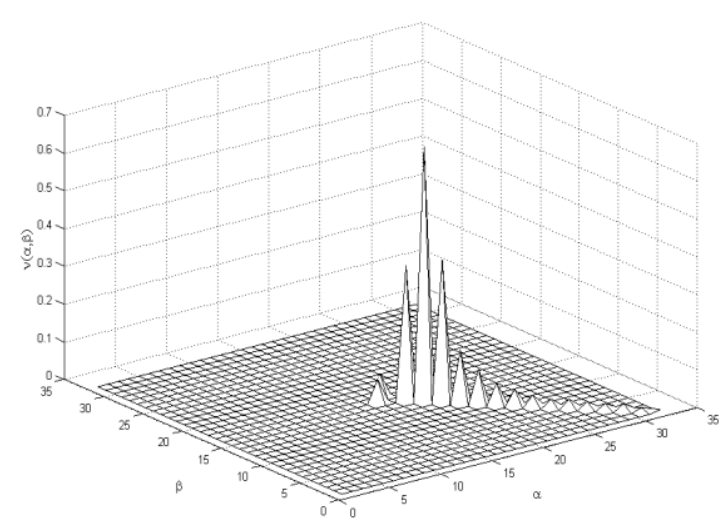

(b)

Fig. 8 Preisach distribution function generated by the proposed method.

3. for $\lambda=3 / 2$ :



(a)

Fig. 9 Experimental measured curve (dotted line) and simulated curve (solid line with marker) generated by the proposed method.

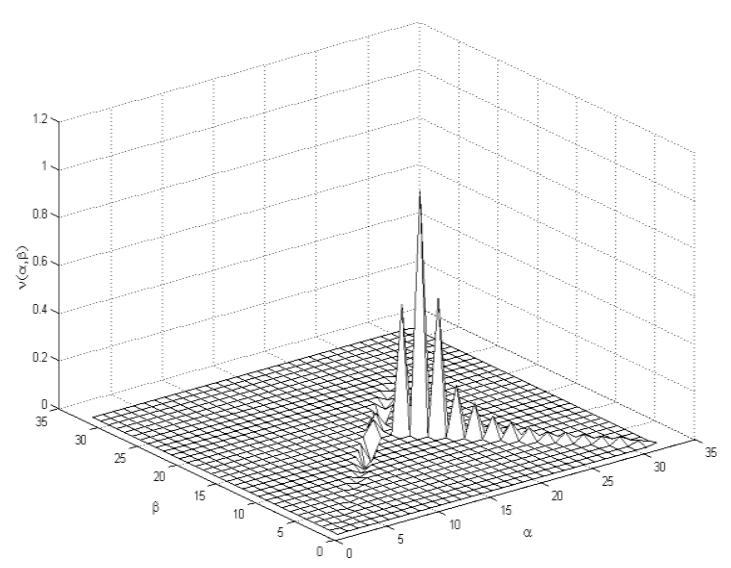

(b)

Fig. 10 Preisach distribution function generated by the proposed method. 
4. For $\lambda=0.01$ :

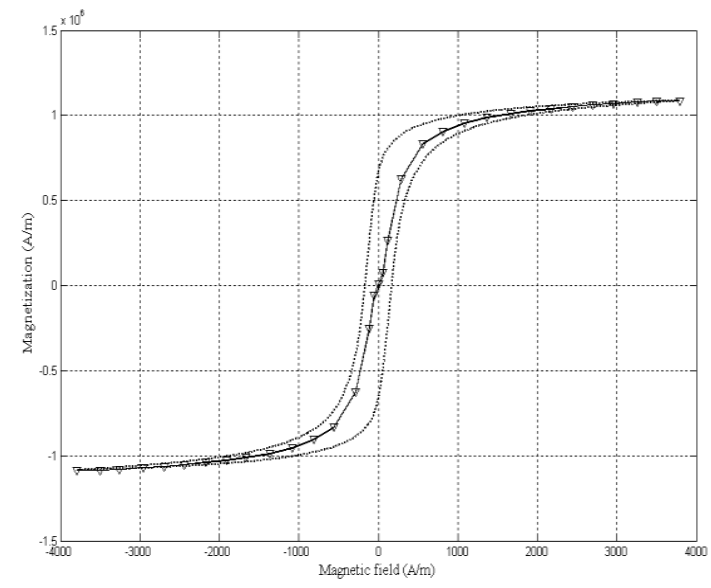

(a)

Fig. 11 Experimental measured curve (dotted line) and simulated curve (solid line with marker) generated by the proposed method.



(b)

Fig. 12 Preisach distribution function generated by the proposed method.

5. For $\lambda=0$ :

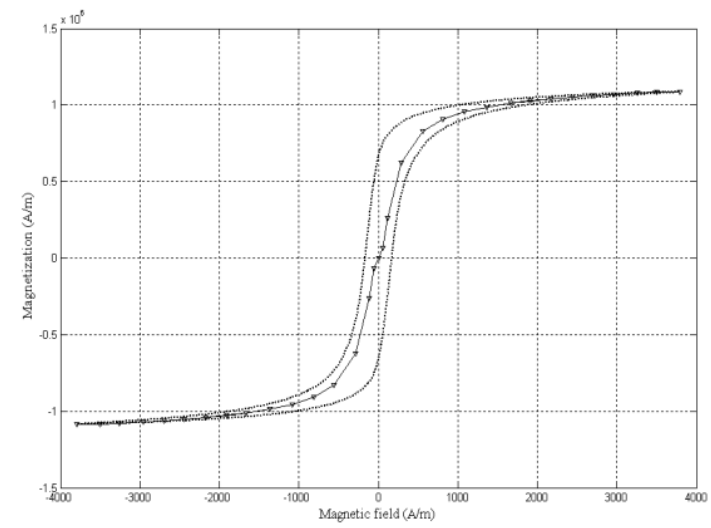

(a)

Fig. 13 Experimental measured curve (dotted line) and simulated curve (solid line with marker) generated by the proposed method.



(b)

Fig. 14 Preisach distribution function generated by the proposed method.

For different values of the factor $\lambda$, we presented the numerically identified Preisach density function and the corresponding hysteresis cycle. The value $\lambda=0.5$ gives a good restitution of the experimental cycle (Fig. 5) and the result is very significant particularly in the zone of the coercive field. The corresponding distribution function is represented in (Fig. 6). For $\lambda=1$ and $\lambda=1.5$, the simulated cycles are not in agreement with the experimental ones (Fig. 6) and (Fig. 9), this is particularly true near the remanence. Finally, when $\lambda \rightarrow 0$ the simulated cycle represents the median of the experimental cycle (Fig. 11) and (Fig. 13) the maximum values of the density function are situated on the line $\alpha=\beta$ which is a significant result because when $\alpha=\beta$ there is no hysteresis (Fig. 12) and Fig. 14).

The $\lambda$ factor is taken equal to the ratio of the magnetization at the bend of saturation $\left(M_{b s}\right)$ to the magnetization of saturation $\left(M_{s}\right)$ in the experimental first magnetization curve. Using the properties of the used material: $\left(M_{b s}\right)=6.319210^{+5}(\mathrm{~A} / \mathrm{m})$ and $\left(M_{s}\right)=1.0866$ $10^{+6}(\mathrm{~A} / \mathrm{m})$, which gives $\lambda=0.5815$. Using this value of the $\lambda$ factor, we obtain a good restitution of the experimental cycle (Fig. 15) and its corresponding distribution function is represented in (Fig. 16):



Fig. 15 Experimental measured curve (dotted line) and simulated curve (solid line with marker) generated by the proposed method with $\lambda=M_{b s} / M_{s}$. 


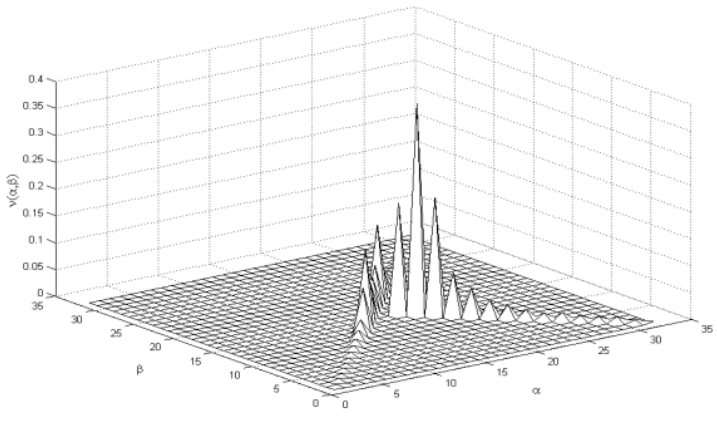

Fig. 16 Preisach distribution function generated by the proposed method with $\lambda=M_{b s} / M_{s}$.

\section{CONCLUSION}

Despite the few experimental data used, the new proposed identification method of the Preisach density function gives very promising results. The method was tested only for the case of a soft magnetic material and the obtained results are very acceptable and allow very good restitution of experimental hysteresis cycles. This technique can be extended to the case of hard magnetic materials by a judicious selection of the $\lambda$ factor. The mentioned advantages make the developed method a powerful numerical tool for Preisach density function identification when only the first magnetization curve is given.

\section{REFERENCES}

[1] MAYERGOYZ, I. D.: Mathematical models of hysteresis, IEEE Trans. Magn, vol. 22, pp. 603-608, 1986.

[2] SZABO, Z. - TUGYI, I. - GY.KADAR, J.FUZI.: Identification procedures for scalar Preisach model, Elsevier Physica B,vol.343, pp.142-147, 2004.

[3] RUDERMAN, M.: Direct recursive identification of the Preisach hysteresis density function, Journal of Magnetism and Magnetic Materials, vol. 348, pp.2226, 2013.

[4] BERTOTTI; G.: Hysteresis in Magnetism, Academic Press, San Diego, 1998, p.480.

[5] RUDERMAN, M.- STROHLA, T.- BERTRAM,T.: Use of Jiles-Atherton and Preisach Hysteresis Models for Inverse Feed-Forward Control, IEEE Trans. Magn, vol. 46, pp.3984-3989, 2010.

[6] RAGUSA, C.: Ananalytical method for the identification of the Preisach distribution function, Journal of Magnetism and Magnetic Materials, vol. 254-255C, pp. 259-261, 2003.
[7] CARDELLI, E. - FIORUCCI, L. - DELLA TORRE, E.: Identification of the Preisach probability functions for soft magnetic materials, IEEE Trans. Magn., vol. 37, pp. 3366-3369,2001.

[8] DAVINO, D. - GIUSTINIANI, A. - VISONE, C.: Fast inverse Preisach models in algorithms for static and quasistatic magnetic-field computations, IEEE Trans. Magn., vol. 44, pp. 862-865, 2008.

[9] RUDERMAN, M.- BERTRAM, M.T.: Identification of soft magnetic B-H characteristics using discrete dynamic Preisach model and a single measured hysteresis loop, IEEE Trans. Magn, vol. 48, pp.1281-1284, 2012.

Received March11, 2014 , accepted September 14, 2014

\section{BIOGRAPHIES}

Leila Chelghoum was born in Algiers (Algeria) in 1970. She received the the "Magister" degree in electrical materials in 2006. In 2012, she joined the Electrical Engineering Institute, University of Batna as an Assistant Professor.

Fatima-Zohra Louai (Dr) was born in Algiers (Algeria) in 1967. She received the engineer degree in electrical engineering from the University of Batna, Algeria in 1990, the DEA degree in electrical engineering from l'INPG de Grenoble (France) in 1991 and the doctorate degree from the University of Nantes (France) in 1995. In 1995, she joined the Electrical Engineering Institute, University of Batna (Algeria), as an Assistant Professor. She was promoted to Associate Professor in 1998. Since 2000, she has been head of the "Modelling of Electromagnetic Phenomena" (MEP) team in LSPIE research laboratory. Her research interests include electromagnetic and thermal phenomena, hysteresis modelling and meshless methods.

Nasreddine Nait-Said (Dr) was born in Batna (Algeria) in 1964. He received the engineer degree in electronics from the "Ecole Nationale Polytechnique", Algiers, (Algeria) in 1985; the "Magister" degree in industrial electricity in 1993 and the doctorate degree in electrical engineering from the University of Batna (Algeria) in 2003. In 1992, he joined the Electrical Engineering Institute, University of Batna as an Assistant Professor. $\mathrm{He}$ was promoted to Associate Professor in 2003. Since 2000, he has been head of the "Artificial Intelligence Techniques Applied to Electrical Systems" (AIT) team in LSPIE research laboratory. His research interests include application of AI techniques and control in the field of electrical machines. 